\title{
Manejo perioperatorio de alteración de la coagulación con coagulograma normal en cirugía de alto riesgo de sangrado
}

\author{
Bellucci P. ${ }^{1}$, Conti M. ${ }^{2}$, Vainstein T. ${ }^{2}$, Mottola M. ${ }^{2}$, Risso J. ${ }^{2}$ \\ 1 HGA Dr. Enrique Tornú, CABA, Argentina. \\ 2 HIGA Dr. Diego Paroissien, Isidro Casanova, Argentina.
}

Introducción: Las principales alteraciones de la coagulación que podrían provocar sangrado intraoperatorio no se encuentran generalmente correlacionadas con alteraciones en el coagulograma (kPTT y TP) solicitado de rutina como evaluación prequirúrgica. El cuestionario HEMSTOP, ha sido desarrollado para detectar la chance de sangrado intraoperatorio. La sensibilidad del coagulograma es del $26,3 \%$ en comparación con la del cuestionario HEMSTOP, del $89,5 \%$. Postulamos este cuestionario como una herramienta de tamizaje sin costo, de fácil implementación y con una tasa de sensibilidad y especificidad igual o mayor que el coagulograma preoperatorio en personas sanas.

Descripción del caso: Paciente femenino de 21 años, sin antecedentes clínicos ni quirúrgicos, que presenta un valor de cuatro respuestas positivas para el cuestionario HEMSTOP. En plan de cirugía para exéresis de tumor cerebral temporal con efecto de masa. Servicio de Hematología informa que frente a los antecedentes de sangrado se pueden suponer alteraciones plaquetarias funcionales o defectos del factor VIII, hallándose tiempo de sangría prolongado. Se sugirió transfusión de plaquetas previa a la cirugía, y disponer de PFC si hubiera sangrado intraoperatorio, también estudio de agregación plaquetaria postalta.

Intraquirúrgico: Monitorización según normas ASA. Profilaxis antibiótica y premedicación. Anestesia general con midazolam, propofol, fentanilo y vecuronio. Acceso femoral derecho y vía arterial radial izquierda. Vías periféricas 14G. Una hora previa a la incisión $1 \mathrm{~g}$ ácido tranexámico, desmopresina $12 \mathrm{mcg}$ y $5 \mathrm{U}$ plaquetas. Previo a sección de duramadre $200 \mathrm{~mL} \mathrm{NaCl}$ hipertónico 3\%, $150 \mathrm{~mL}$ manitol 10\% y furosemida $20 \mathrm{mg}$. Se extrae muestra basal de gases arteriales, ionograma y hemoglobina. Mantenimiento con sevofluorano y remifentanilo. Se utilizó noradrenalina 0,02-0,1 $\mathrm{mcg} / \mathrm{kg} / \mathrm{min}$. Sangrado intraoperatorio $1.000 \mathrm{~mL}$, se transfundieron 5 U PFC. Corrección del déficit de calcio. Diuresis $1 \mathrm{~mL} / \mathrm{kg} /$ hora. Analgesia postoperatoria tramadol $50 \mathrm{mg}$. Al despertar lúcida, Glasgow 15/15, sin signos de foco motor/sensitivo, hemodinámicamente estable, sin necesidad de vasoactivos. Postoperatorio en UTI sin intercurrencias.

Comentarios y Discusión: La baja sensibilidad de las pruebas disponibles para tamizar trastornos de la coagulación en pacientes sanos genera la necesidad de buscar nuevos métodos para su screening. Sin una herramienta que medie esta circunstancia, cirugías de alto riesgo de sangrado pueden conllevar un aumento en la morbimortalidad de estos pacientes; con el aumento de costo en salud pública de todo evento crítico.

Posteriormente a esta experiencia, los residentes decidimos realizar la puesta a prueba del HEMSTOP en los preanestésicos de nuestros pacientes programados, proponiéndolo como una herramienta para la evaluación de los trastornos de la coagulación con mejor predicción del sangrado intraoperatorio.

https://doi.org/10.25237/congresoclasa2019.63 